\title{
Electrochemical Deposition of a Potassium Salt Using Crown Ether with a Ferrocenyl Moiety
}

\author{
Yoshiyuki ISHII and Tetsuo SAJI*
}

\author{
Received June 14, 1996 ; Accepted July 17, 1996
}

\section{INTRODUCTION}

In recent years redox-switched molecular aggregates have been extensively studied. For example, redox switched complexes of a crown ether with ions, surfactants, ${ }^{2}$ and vesicles ${ }^{3)}$ have been reported. Recently, we reported electrochemical ion transport using a redox active neutral crown ether ${ }^{4}$ and electrochemical ${ }^{\text {s }}$ and electroless ${ }^{6}$ formations of organic thin films using redox active surfactants. In this paper, a novel method for the deposition of a potassium salt is demonstrated using the follwong crown ether (1):

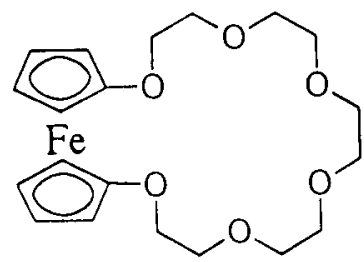

1

\section{EXPERIMENTAL}

\subsection{Reagents}

$1,4,7,10,13,16$-Hexaoxa [16] ferrocenophane (1) was prepared by a previously reported method ${ }^{\text {t }}$. Potassium picrate ( $\mathrm{K}^{+} \mathrm{PIC}$ ) was used as the potassium salt which was not dissolved in dichloromethane. On the other hand, in the presence of 1 , this salt was dissolved in dichloromethane. Tetra- $n$-butylammonium picrate (TBA PIC) was prepared by the literature method $^{\text {B) }}$ and was used as the supporting electrolyte.

\subsection{Apparatus and Methods}

Cyclic voltammetry was performed using a homemade potentiostat with circuitry for the compensation of solution resistance. A platinum sphere was used as the working electrode. $\mathrm{An} \mathrm{Ag} / \mathrm{AgNO}_{3}$ electrode and a platinum wire were used as the reference and counter electrode, respectively. An indium tin oxide (ITO, 10 $\Omega /$ square) electrode was used as the working elect-

Department of Chemical Engineering. Tokyo Institute of Technology (Ohokayama 2-12-1, Meguro-ku, Tokyo 152)

Key words: Crown Ether, Ferrocene, Potassium Salt, Deposition rode for the controlled-potential electrolysis. Controlled-potential electrolysis of the solution at the ITO electrode was done while stirring the solution. After a definite amount of electricity $(q)$ had passed through the ITO electrode, the electrode was rinsed with dichloromethane and dried in air. All electrochemical measurements were carried out at $25^{\circ} \mathrm{C}$ under a nitrogen atmosphere.
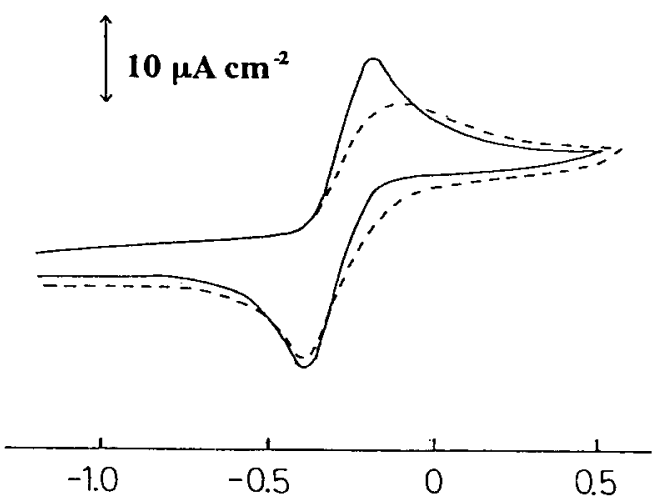

$\mathrm{E} / \mathrm{V}$ vs. Ag/AgNO

Fig. 1 Cyclic voltammograms of dichloromethane solutions containing $2.3 \mathrm{mM} 1$ and $0.1 \mathrm{M} \mathrm{TBA}^{+} \mathrm{PIC}$ without $\mathrm{K}^{+} \mathrm{PIC}{ }^{-}(-)$and with $\mathrm{K}^{+} \mathrm{PIC}^{-}$(saturated) $\left(-\rightarrow\right.$. Scan rate: $50 \mathrm{mVs}^{-1}$.

\section{RESULTS AND DISCUSSION}

Figure 1 shows the cyclic voltammograms of a dichloromethane solution containing $2.3 \mathrm{mM} 1$ and 0. $1 \mathrm{M} \mathrm{TBA}^{+} \mathrm{PIC}^{-}$without $\mathrm{K}^{+} \mathrm{PIC}^{-}$and with $\mathrm{K}^{+} \mathrm{PIC}$ (saturated). In the absence of potassium ion, 1 exhibited a one-electron oxidation step with a half-wave potential of $-0.33 \mathrm{~V}$ vs. $\mathrm{Ag} / \mathrm{AgNO}_{3}$. When the solution was saturated with $\mathrm{K}^{+} \mathrm{PIC}$, this oxidation peak potential was shifted in the positive direction by approximately $150 \mathrm{mV}$. This positive shift due to the addition of potassium ion reflects the abrupt decrease in metal ion binding of $1^{+}$by the oxidation of 1 . The binding strength of $\mathbf{1}^{+}$may be much weaker than that of 1 . The difference in stability constants is calculated as follows. ${ }^{1)}$

$$
\begin{aligned}
& \begin{array}{lll}
\mathbf{1}^{+}+\mathrm{e} & \rightleftarrows \mathbf{1} & E_{\mathrm{o}}^{\mathrm{f}} \\
\mathbf{1}^{+} \cdot \mathrm{K}^{+}+\mathrm{e} & \rightleftarrows \mathbf{1} \cdot \mathrm{K}^{+} & E_{\mathrm{o}}^{\mathrm{c}}
\end{array} \\
& E_{\mathrm{o}}{ }^{\mathrm{c}}-E_{\mathrm{o}}^{\mathrm{f}}=(R T / \mathbf{n} F) \ln \left(K_{\mathrm{R}} / K_{\mathrm{O}}\right)
\end{aligned}
$$


where $K_{\mathrm{R}}\left\{=\left[1 \cdot \mathrm{K}^{+}\right] /[1]\left[\mathrm{K}^{+}\right]\right\}$and $K_{\mathrm{O}}$ $\left\{=\left[\mathbf{1}^{+} \cdot \mathrm{K}^{+}\right] /\left[\mathrm{1}^{+}\right]\left[\mathrm{K}^{+}\right]\right\}$are the stability constants of 1 and $1^{+}$with metals, respectively. The other symbols have their usual meanings. In this case, only the oxidation peak potential shifted. No shift in the reduction peak was observed. This may be explained by the following hypothesis: the oxidation of $1 \cdot \mathrm{K}^{+}$ proceeds according to reaction (2), however, once 1 . $\mathrm{K}^{+}$is oxidized, most of the ions binding to 1 are completely dissociated to $\mathrm{K}^{+}$and $1^{+}$. No shift in the reversal peak potential may be due to the slow reaction between regenerated 1 and $\mathrm{K}^{+}$. The ratio of the stability constants is calculated using equation (3) as 340 . This value suggests that the $1 \cdot \mathrm{K}^{+}$complex completely dissociates to $\mathrm{K}^{+}$and $\mathrm{1}^{+}$by electrochemical oxidation of this complex at the ITO electrode.

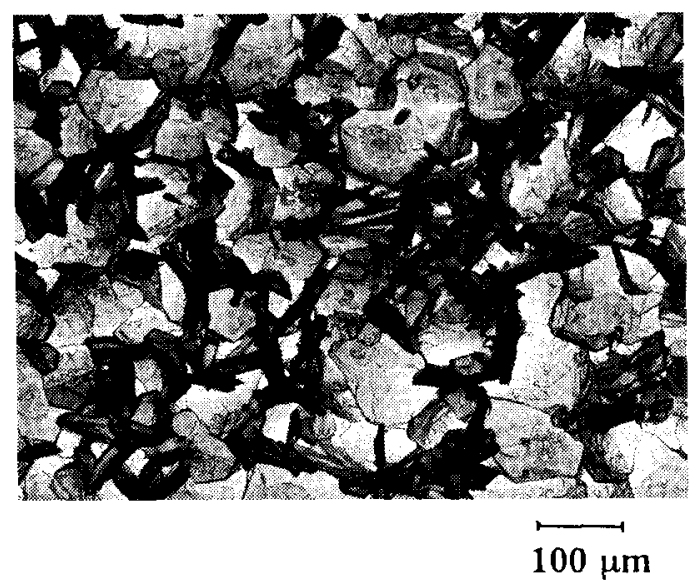

Fig. 2 Micrograph of the film on the ITO obtained by controlled-potential electrolysis of a dichloromethane solution containing $4 \mathrm{mM} 1,0.2 \mathrm{M} \mathrm{TBA}^{+} \mathrm{PIC}$, and saturated $\mathrm{K}^{+} \mathrm{PIC}$ at $+0.80 \mathrm{~V}$ vs. $\mathrm{Ag} / \mathrm{AgNO}_{3}$ while stirring the solution. $q: 0.50$ coulomb cm .

Controlled potential electrolysis of a dicholomethane solution containing $4 \mathrm{mM} 1,0.2 \mathrm{M} \mathrm{TBA}^{+} \mathrm{PIC}^{-}$and saturated $\mathrm{K}^{+} \mathrm{PIC}$ ' was done at $+0.80 \mathrm{~V}$ vs. $\mathrm{Ag} / \mathrm{AgNO}_{3}$ while stirring the solution. A yellow film was formed on the ITO electrode by this electrol ysis. Figure 2 shows the micrograph of the film on the ITO electrode, which indicates that this film is composed of crystallites. The electronic absorption spectrum of an aqueous solution prepared by washing this film was the same as that of potassium picrate and no absorption peaks of 1 and $\mathbf{1}^{+}$ were observed. This result shows the film formed on the ITO electrode is made of $\mathrm{K}^{+} \mathrm{PIC}$.

Figure 3 is a plot of the amount of $\mathrm{K}^{+} \mathrm{PIC}$ deposited on the ITO electrode vs. amount of electricity, which shows linear relationship. The average current efficiency of the series of values in Fig. 3 is $56 \%$. This film

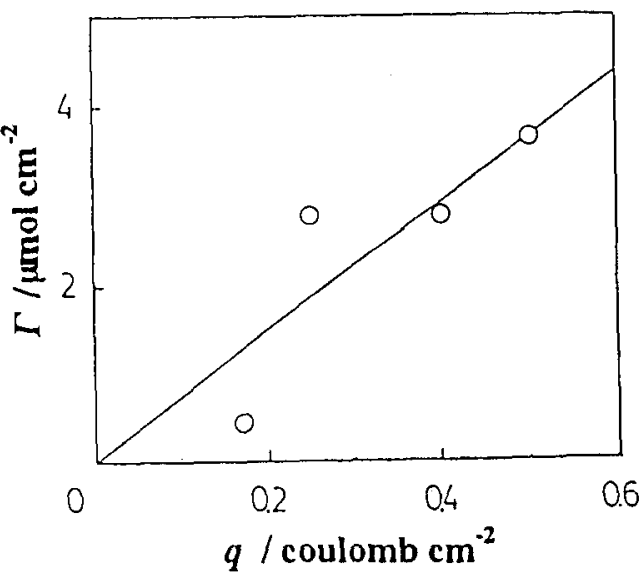

Fig. 3 Amount of $\mathrm{K}^{+} \mathrm{PIC}^{-}(I)$ vs. the amount of electricity passed through the TTO electrode $(q)$.

formation may be explained by the following processes: (1) $1 \cdot \mathrm{K}^{+}$complex diffuses to the ITO electrode surface. (2) 1 is oxidized to $1^{+}$. (3) $\mathrm{K}^{+}$is released from 1 due to the enhancement in electrostatic repulsion between the positively charged ferrocenyl moiety of $\mathrm{1}^{+}$and $\mathrm{K}^{+}$. (4) $\mathrm{K}^{+} \mathrm{PIC}-1$ is supersaturated and finally deposit on the ITO electrode.

\section{CONCLUSION}

The present experiments show that the use of the redox active crown ether 1 makes it possible to deposit a potassium salt with electrolysis.

\section{REFERENCES}

1) A. Kaifer, D. A. Gustowski, L. Echegoyen, V. J. Gatto, R. A. Cleary, P. T. Morgan, C. R. Morgan,D. M. Goli, A. M. Goli, A. M. Rios and G. W. Gokel, J. Am. Chem. Soc., 107, 1958 (1985); (b) T. Saji, Chem. Lett., 1986, 275.

2) T. Saji, K. Hoshino and S. Aoyagui, J. Am. Chem. Soc., 107, 6865 (1985).

3) J. C. Medina, I. Gay, Z. Chen, L. Echegoyen and G. W. Gokel, J. Am. Chem. Soc., 113, 365 (1991).

4) T. Saji and J. Kinoshita, J. Chem. Soc., Chem. Commun., 1986, 716.

5) K. Hoshino and T. Saji, J. Am. Chem. Soc., 107, 6865 (1985); T. Saji, K. Hoshino, Y. Ishii and M. Goto, J. Am. Chem. Soc., 113, 450 (1991).

6) T. Saji, K. Ebata, K. Sugawara, S. Liu and K. Kobayashi, J. Am. Chem. Soc., 116, 6053 (1994).

7) S. Akabori, Y. Habata, Y. Sakamoto, M. Sato and S. Ebine, Bull. Chem. Soc. Jpn., 56, 537 (1983).

8) C. M. French and D. M. Muggleton, J. Chem. Soc., $1957,2131$. 